[23] M. M. Ilić, A. Ž. Ilić, and B. M. Notaroš, "Higher order large-domain FEM modeling of 3-D multiport waveguide structures with arbitrary discontinuities," IEEE Trans. Microw. Theory Tech., vol. 52, no. 6, pp. 1608-1614, Jun. 2004.

[24] R. D. Graglia and G. Lombardi, "Singular higher order complete vector bases for finite methods," IEEE Trans. Antennas Propag., vol. 52, pp. 1672-1685, Jul. 2004

[25] M. M. Ilić and B. M. Notaroš, "Higher order large-domain hierarchical FEM technique for electromagnetic modeling using Legendre basis functions on generalized hexahedra," Electromagn., vol. 26, pp. 517-529, Oct. 2006.

[26] B. M. Notaroš, "Higher order frequency-domain computational electromagnetics," IEEE Trans. Antennas Propag. Special Issue on Large and Multiscale Comput. Electromagn., , vol. 56, no. 8, pp. 2251-2276, Aug. 2008.

[27] M. Djordjević and B. M. Notaroš, "Double higher order method of moments for surface integral equation modeling of metallic and dielectric antennas and scatterers," IEEE Trans. Antennas Propag., vol. 52, no. 8, pp. 2118-2129, Aug. 2004.

[28] M. M. Ilić, M. Djordjević, A. Ž. Ilić, and B. M. Notaroš, "Higher order hybrid FEM-MoM technique for analysis of antennas and scatterers," IEEE Trans. Antennas Propag., to be published.

\section{Extended Analysis of Retrodirective Cross-Eye Jamming}

W. P. du Plessis, J. W. Odendaal, and J. Joubert

\begin{abstract}
An extended and rigorous analysis of retrodirective cross-eye jamming in a radar system scenario is presented. This analysis removes the approximations that limit the validity of other analyses of cross-eye jamming. These results imply that under certain conditions, a monopulse radar system can be more easily deceived than suggested by conventional cross-eye analyses. Furthermore, the cross-eye jammer antenna patterns do not affect the induced monopulse error.
\end{abstract}

Index Terms-Electronic warfare, radar countermeasures, radar tracking.

\section{INTRODUCTION}

Cross-eye jamming is an electronic warfare (EW) technique that induces an angular deception in a radar system by recreating the worstcase glint angular error [1]-[6]. The target angular error is created by transmitting out of phase signals from two or more onboard antennas, thereby deceiving the radar into believing that the target is spatially removed from its true position.

In analyzing the performance of cross-eye jamming on a radar system, the effect of cross-eye jamming is generally considered as a distortion of the phase front incident on the antenna(s) of the radar system [2]-[5] or a change in the direction of the incident Poynting vector [7]. In [8], Kajenski has shown that the phase-front and Poynting vector approaches to analyzing cross-eye jamming are equivalent. Cross-eye jamming has also been analyzed using first-order Taylor

Manuscript received October 10, 2008; revised February 13, 2009. First published July 10, 2009; current version published September 02, 2009.

W. P. du Plessis is with the University of Pretoria, Pretoria 0002, South Africa and also with the Council for Scientific and Industrial Research (CSIR) - Defence, Peace, Safety and Security (DPSS), Pretoria Gauteng 0001, South Africa (e-mail: wduplessis@csir.co.za).

J. W. Odendaal and J. Joubert are with the Centre for Electromagnetism, University of Pretoria, Pretoria 0002, South Africa

Digital Object Identifier 10.1109/TAP.2009.2027353

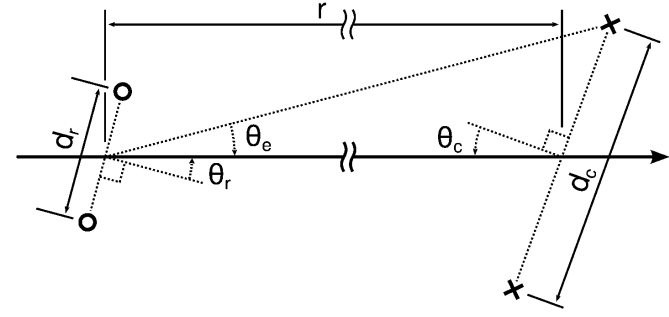

Fig. 1. The geometry used for the cross-eye jammer derivation.

approximations to either the sum- and difference-channel antenna patterns [9], [10] or the lobes of an amplitude-comparison tracking radar system [11]. Recently, a graphical vector representation of the fields incident on a radar antenna due to cross-eye jamming was used to analyze cross-eye jamming performance [6], [12].

These analyses assume (either directly or indirectly through the way the antenna patterns are determined) that amplitude variations over the radar antenna aperture are negligible. Another significant limitation of conventional cross-eye analyses is that they ignore the retrodirective implementation of most cross-eye systems [2], [3], [5], [6]. While retrodirective (Van Atta) arrays have been extensively considered in the literature (e.g., [13]-[19]), the work has concentrated on the issues like mutual coupling and mismatches in the array itself rather than its effects on a radar.

This communication presents the summarized results of an extended and rigorous analysis of cross-eye jamming in a monopulse radar system scenario that has recently been performed [20]. The extended analysis takes into consideration the physical separation between the antennas of a monopulse radar system and derives an expression for the induced angular error due to the cross-eye jammer. Induced angular error results that demonstrate the differences between the conventional and extended cross-eye jamming analyses are presented.

Section II outlines the derivation of the extended analysis. Section III presents a comparison between induced angular errors obtained with the classical and extended analyses, and also considers some of the more significant implications of the extended analysis. Finally, Section IV provides a brief conclusion.

\section{THEORY}

The purpose of cross-eye jamming is to produce an angular error in a radar system, thus causing the radar to believe that the target is spatially removed from its true position. Consider the cross-eye jamming scenario shown in Fig. 1. Assume the phase-comparison monopulse radar consists of two identical antennas separated by a distance of $d_{r}$ (denoted by the circles in Fig. 1). The cross-eye elements have a linear separation of $d_{c}$ at a range of $r$ giving an angular separation of $2 \theta_{e}$ from the radar's perspective (denoted by the crosses in Fig. 1). The directions to the top and bottom cross-eye antennas are thus $\theta_{r} \pm \theta_{e}$ respectively.

While amplitude-comparison monopulse systems are not explicitly considered in this correspondence, Sherman [10] shows that amplitudeand phase-comparison monopulse systems are equivalent.

The underlying principle of cross-eye jamming is that the radar is not able to resolve the individual jammer antennas and therefore responds to their combined effect. This implies that the cross-eye jammer system is in the far field of the radar antenna $\left(r \gg d_{r}\right)$. However, it is assumed that although the radar is in the far field of the individual jammer antenna elements, it is not in the far field of the complete cross-eye jammer system [6], [12]. 
The fact that a cross-eye jammer transmits signals that are out of phase means that the signal received by the sum channel of the radar will be very small. If it is further assumed that the radar is in the far field of the cross-eye jammer system, there will be no amplitude or phase variations over the receiving radar aperture. Under these conditions, the signal received by both the sum and difference channels of the monopulse radar will be negligible, and the cross-eye jammer will have no effect on the radar. Hence the assumption that the radar is not in the far field of the cross-eye jammer system, although it is in the far field of the individual jammer antenna elements.

The value of $\theta_{e}$ can be determined from

$$
\theta_{e}=\frac{d_{c} / 2 \cos \left(\theta_{c}\right)}{r \pm d_{c} / 2 \sin \left(\theta_{c}\right)} \approx \frac{d_{c}}{2 r} \cos \left(\theta_{c}\right)
$$

because $r$ is much greater than $d_{c}$. Given that $r$ and $d_{c}$ are usually in the order of hundreds of meters to kilometers and meters respectively, the error caused by the approximation in (1) is typically much less than $1 \%$.

A retrodirective cross-eye jammer is assumed, which means that the entire signal received at one jammer element is retransmitted at the other jammer element after some delay. The main effect of the retrodirective assumption is that the path lengths between the radar and the cross-eye jammer elements cancel out because all signals travel along the same path, just in different directions.

When the angle between the direction of the main beam of the radar and the center of the cross-eye jammer is $\theta_{r}$ as shown in Fig. 1, the normalized sum and difference patterns of the radar antenna in the directions of the top and bottom cross-eye jammer elements can be derived using array theory [21] to give

$$
S_{1,2}=P_{r}\left(\theta_{r} \pm \theta_{e}\right) \cos \left[\beta \frac{d_{r}}{2} \sin \left(\theta_{r} \pm \theta_{e}\right)\right]
$$

and

$$
D_{1,2}=P_{r}\left(\theta_{r} \pm \theta_{e}\right) \sin \left[\beta \frac{d_{r}}{2} \sin \left(\theta_{r} \pm \theta_{e}\right)\right]
$$

where $P_{r}(\theta)$ is the pattern of the elements used to form the phasecomparison monopulse array.

By noting that $\theta_{e}$ is very small, defining

$$
k=\beta \frac{d_{r}}{2} \sin \left(\theta_{r}\right)
$$

and

$$
k_{c}=\beta \frac{d_{r}}{2} \cos \left(\theta_{r}\right) \theta_{e}
$$

and performing some elementary manipulations, the sum- and difference-channel gains can be rewritten as

$$
S_{1,2}=P_{r}\left(\theta_{r} \pm \theta_{e}\right) \cos \left(k \pm k_{c}\right)
$$

and

$$
D_{1,2}=P_{r}\left(\theta_{r} \pm \theta_{e}\right) \sin \left(k \pm k_{c}\right) .
$$

Assuming that the signal that passes through the cross-eye jammer from antenna 2 to antenna 1 has an amplitude gain of $a$ and a phase shift of $\phi$ relative to the signal that passes from antenna 1 to antenna 2 of the jammer, the signal received by the radar in the sum channel will be

$$
\begin{aligned}
S_{r}= & S_{1} P_{c}\left(\theta_{c}+\theta_{e}\right) S_{2} P_{c}\left(\theta_{c}-\theta_{e}\right) \\
& +a e^{j \phi} S_{2} P_{c}\left(\theta_{c}-\theta_{e}\right) S_{1} P_{c}\left(\theta_{c}+\theta_{e}\right) \\
= & P_{r}\left(\theta_{r}-\theta_{e}\right) P_{c}\left(\theta_{c}-\theta_{e}\right) P_{r}\left(\theta_{r}+\theta_{e}\right) P_{c}\left(\theta_{c}+\theta_{e}\right) \\
& \times \frac{1}{2}\left(1+a e^{j \phi}\right)\left[\cos (2 k)+\cos \left(2 k_{c}\right)\right]
\end{aligned}
$$

and the signal received by the radar in the difference channel will be

$$
\begin{aligned}
D_{r}= & S_{1} P_{c}\left(\theta_{c}+\theta_{e}\right) D_{2} P_{c}\left(\theta_{c}-\theta_{e}\right) \\
& +a e^{j \phi} S_{2} P_{c}\left(\theta_{c}-\theta_{e}\right) D_{1} P_{c}\left(\theta_{c}+\theta_{e}\right) \\
= & P_{r}\left(\theta_{r}-\theta_{e}\right) P_{c}\left(\theta_{c}-\theta_{e}\right) P_{r}\left(\theta_{r}+\theta_{e}\right) P_{c}\left(\theta_{c}+\theta_{e}\right) \\
& \times \frac{1}{2}\left[\left(1+a e^{j \phi}\right) \sin (2 k)+\left(1-a e^{j \phi}\right) \sin \left(2 k_{c}\right)\right]
\end{aligned}
$$

where $P_{c}\left(\theta_{c} \pm \theta_{e}\right)$ are the gains of the top and bottom cross-eye jammer antennas in the direction of the radar.

An exact monopulse processor forms its error signal by dividing the difference-channel return in (11) by the sum-channel return in (9) and taking the real part of the result [10] giving

$$
\Re\left\{\frac{D_{r}}{S_{r}}\right\}=\frac{\sin (2 k)+\sin \left(2 k_{c}\right) \frac{1-a^{2}}{1+a^{2}+2 a \cos (\phi)}}{\cos (2 k)+\cos \left(2 k_{c}\right)} .
$$

The monopulse error is then calculated using (13) [10].

The monopulse error for a single scatterer is [10]

$$
\Re\left\{\frac{D_{r}}{S_{r}}\right\}=\tan (k)
$$

and it can be shown that (12) reduces to this form when the separation between the cross-eye jammer antennas tends to zero $\left(d_{c} \rightarrow 0\right.$ so $k_{c} \rightarrow$ $0)$.

\section{RESULTS AND DISCUSSION}

The result for the monopulse error given in (12) has the same form as the following approximate result given by Meade [9] and Sherman [10]

$$
\Re\left\{\theta_{i}\right\} \approx \theta_{r}-\theta_{e} \frac{1-a^{2}}{1+a^{2}+2 a \cos (\phi)} .
$$

The error obtained using other glint analyses is the same as that in (14), [7], [22], [23], though the arctangent of the error term should be used [24]. This similarity led Neri to define a cross-eye gain as the error term divided by $\theta_{e}$ giving [3]

$$
G_{C}=\frac{1-a^{2}}{1+a^{2}+2 a \cos (\phi)}
$$

and this cross-eye gain appears in (12).

It can be shown that (12) reduces to (14) when $\theta_{r}$ and $\theta_{c}$ are small. The only difference is the sign of the second term, and is due to the definition of the directions of $\theta_{r}$ and $\theta_{e}$.

The indicated angles from (12) (using (13) and (4) to convert the monopulse error to an indicated angle) and (14) are plotted against radar angle $\left(\theta_{r}\right)$ in Fig. 2 for the following parameters typical of a missile threat against a ship or aircraft:

- $10^{\circ}$ radar beamwidth $\left(d_{r}=2.54\right.$ wavelengths, and each radar element is a linear source 2.54 wavelengths long);

- $1 \mathrm{~km}$ jammer range $(r=1 \mathrm{~km})$;

- $10 \mathrm{~m}$ jammer element separation $\left(d_{c}=10 \mathrm{~m}\right)$;

- $30^{\circ}$ jammer rotation $\left(\theta_{c}=30^{\circ}\right)$;

- $0.5 \mathrm{~dB}$ jammer amplitude mismatch $(\mathrm{a}=0.9441)$;

- the relative phases of the two directions through the jammer $(\phi)$ for each curve are indicated in Fig. 2.

The vertical lines in Fig. 2 at $\pm 11.1^{\circ}$ and $\pm 11.6^{\circ}$ are caused by a $180^{\circ}$ phase shift in the sum-channel return at nulls. The trigonometric functions in (4) and (5) cause the monopulse error to repeat. 


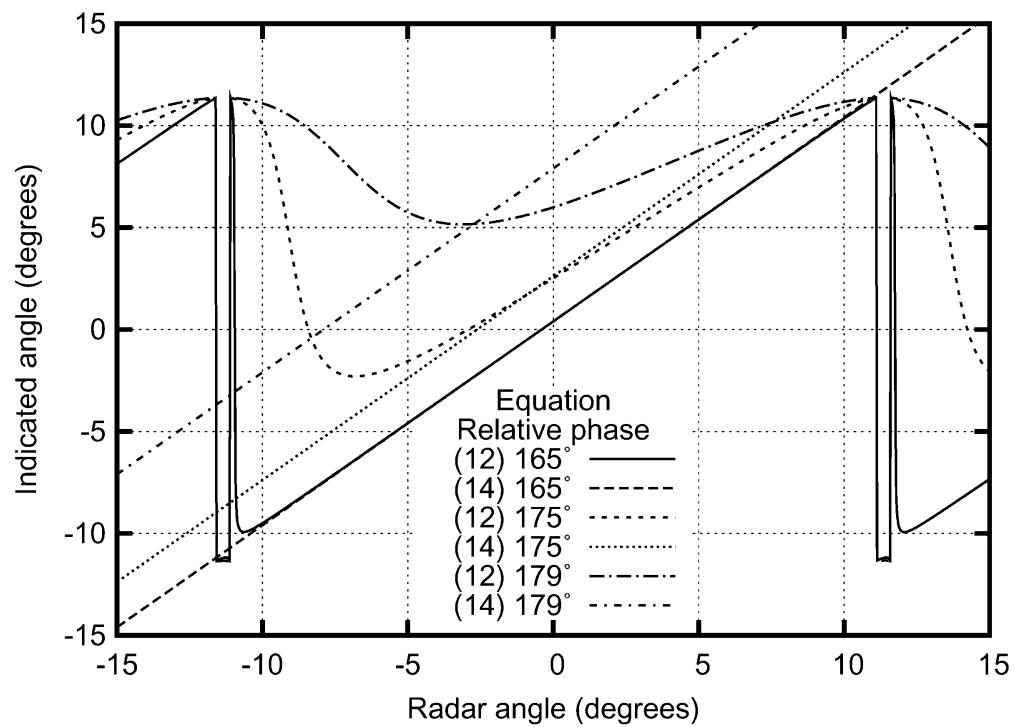

Fig. 2. The monopulse error for the conditions described in the text.

The curves in Fig. 2 corresponding to $165^{\circ}$ and $175^{\circ}$ relative phase shifts between the two directions through the cross-eye jammer system are seen to have more than one zero near the origin. Only the zeros nearest the origin are stable because the sign of the monopulse error will tend to drive the radar away from the other zeros.

Equation (14) was derived under the assumption that there is no significant amplitude variation over the radar antenna aperture, so only phase variations need to be considered. This assumption is very accurate when the relative phase shift of the two directions through the cross-eye jammer system is far from $180^{\circ}$, but becomes increasingly poor as the relative phase shift approaches $180^{\circ}$. The agreement between (12) and (14) is seen to follow a similar trend, reflecting the inaccuracies caused by ignoring amplitude variations.

The use of a retrodirective cross-eye jammer means that all paths from the radar to the jammer and back are the same length as long as it can be assumed that the jammer is in the far field of the radar antenna. Under this approximation, the results in (9), (11) and (12) are exact.

Changes in $a$ and $\phi$ do not affect the shape of the sum-channel return in (9) as $\theta_{r}$ and $\theta_{c}$ vary, but rather cause amplitude scaling and phase offsets that are independent of angle. In the case of a perfect cross-eye jammer, $a \rightarrow 1$ and $\phi \rightarrow 180^{\circ}$ giving a monopulse sum-channel return that tends to zero for all values of $\theta_{r}$ and $\theta_{c}$ (though the monopulse difference-channel return is large under these conditions). This is an extremely interesting result because it means that a radar that uses the same antenna pattern for transmission and reception (e.g., a conical-scan radar) will not receive a return from a perfect retrodirective cross-eye jammer.

The shape of the difference-channel return in (11) as a function of $\theta_{r}$ and $\theta_{c}$ is strongly dependent on $a$ and $\phi$. The first term in (11) depends on $1+a e^{j \phi}$ and becomes zero when the radar antenna points exactly between the two cross-eye elements because of the $\sin \left(\theta_{r}\right)$ term in (4). The second term in (11) depends on $1-a e^{j \phi}$, has a maximum when the radar antenna points exactly between the cross-eye jammer elements due to the $\cos \left(\theta_{r}\right)$ term in (5), and tends to increase as the jammer element separation is increased as a result of the $\theta_{e}$ term in (5). The first term in (11) is maximized and the second minimized in a retrodirective array ( $a \rightarrow 1$ and $\phi \rightarrow 0$ ), so the difference-channel return will be minimized when the radar points exactly between the array elements, as expected. The converse is true for a cross-eye jammer $(a \rightarrow 1$ and $\phi \rightarrow 180^{\circ}$ ), and this means that a radar will receive a large differencechannel return when it points exactly between the jammer elements.
A cross-eye jammer will thus produce a large error when a monopulse radar is pointing exactly between the elements of the jammer because the jammer causes the radar to receive a small sum-channel return and a large difference-channel return. That said, the true situation is slightly more complex because an exact monopulse processor only uses the real part of the monopulse error (12), so only that portion of the difference-channel return which is in phase with the sum-channel return is considered.

An interesting characteristic of the $179^{\circ}$ case in Fig. 2 is that the monopulse error never becomes zero (the vertical lines due to the nulls in the sum pattern connect two nonzero values of differing signs) while (14) predicts an error of $7.9^{\circ}$. This suggests that a monopulse radar can be rotated away from a target more easily than suggested by (14) when $a$ is close to 1 and $\phi$ is close to $180^{\circ}$.

The most important observation about the monopulse error in (12) is that the element patterns $P_{r}(\theta)$ and $P_{c}(\theta)$ cancel and thus have no effect on the induced angular error. Thus, while increasing the gain of the antennas in a cross-eye jammer will increase the strength of the signals received by a radar as shown in (9) and (11), it will not increase the monopulse error induced in the radar.

\section{CONCLUSION}

An extended and rigorous analysis of cross-eye jamming in a monopulse radar system scenario was performed. Results of induced angular error that demonstrate the difference between conventional and the extended cross-eye jamming analyses were presented. The implications of these results include the observations that the cross-eye jammer antenna element patterns do not affect the induced monopulse error, and the fact that there are cases for which the induced monopulse error will not be zero at any angle, which implies that the monopulse radar system can be deceived more easily than suggested by conventional analyses.

\section{ACKNOWLEDGMENT}

W. du Plessis would like to thank the Armaments Corporation of South Africa (Armscor) for granting permission to publish this work in support of his studies.

\section{REFERENCES}

[1] B. L. Lewis and D. D. Howard, "Security Device," U.S. Patent 4006 478, Feb. 1, 1977. 
[2] L. B. Van Brunt, Applied ECM. New York: EW Engineering, Inc., 1978, vol. 1.

[3] F. Neri, Introduction to Electronic Defense Systems. Boston: Artech House, 1991.

[4] C. Musso and C. Curt, "Robustness of a new angular countermeasure," in Proc. Radar 97, Oct. 14-16, 1997, pp. 415-419.

[5] N. M. Harwood, W. N. Dawber, V. A. Kluckers, and G. E. James, "Multiple-element crosseye," IET Radar Sonar Navig., vol. 1, no. 1, pp. 67-73, Feb. 2007.

[6] L. Falk, "Cross-eye jamming of monopulse radar," in Proc. IEEE Waveform Diversity Design Conf., Jun. 4-8, 2007, pp. 209-213.

[7] J. H. Dunn and D. D. Howard, "Radar target amplitude, angle, and doppler scintillation from analysis of the echo signal propagating in space," IEEE Trans. Microw. Theory Tech., vol. 9, no. 9, pp. 715-728, Sept. 1968.

[8] P. Kajenski, "Comparison of two theories of angle glint: Polarization considerations," IEEE Trans. Aerosp. Electron. Syst., vol. 42, no. 1, pp. 206-210, Jan. 2006.

[9] J. E. Meade, , A. S. Locke, Ed., "Target considerations," in Guidance. Princeton, NJ: D. Van Nostrand, 1955, pp. 435-444, ch. 11.

[10] S. M. Sherman, Monopulse Principles and Techniques. Boston: Artech House, 1984.

[11] Y. Stratakos, G. Geroulis, and N. Uzunoglu, "Analysis of glint phenomenon in a monopulse radar in the presence of skin echo and non-ideal interferometer echo signals," J. Electromagn. Waves Applicat., vol. 19, no. 5, pp. 697-711, 2005.

[12] L. Falk, C. Arvidsson, S. Berglund, and A. Eneroth, "Simple derivation of crosseye jamming principles," in Proc. MilTech 2 Conf., Oct. 25-26, 2005, pp. 93-100.

[13] L. C. Van Atta, "Electromagnetic Reflector," U.S. Patent 2908 002, Oct. 6, 1959.

[14] E. Sharp and M. Diab, "Van Atta reflector array," IEEE Trans. Antennas Propag., vol. 8, no. 4, pp. 436-438, Jul. 1960.

[15] J. Appel-Hansen, "A Van Atta reflector consisting of half-wave dipoles," IEEE Trans. Antennas Propag., vol. 14, no. 6, pp. 694-700, Nov. 1966.

[16] T. Larsen, "Reflector arrays," IEEE Trans. Antennas Propag., vol. 14, no. 6, pp. 689-693, Nov. 1966.

[17] E. Nielsen, "Square Van Atta reflector with conducting mounting plane," IEEE Trans. Antennas Propag., vol. 18, no. 1, pp. 48-54, Jan. 1970.

[18] B. D. Steinberg, Principles of Aperture and Array System Design: Including Random and Adaptive Arrays. New York: Wiley-Interscience, 1976.

[19] S. Chung and K. Chang, "A retrodirective microstrip antenna array," IEEE Trans. Antennas Propag., vol. 46, no. 12, pp. 1802-1809, Dec. 1998.

[20] W. P. du Plessis, Electronic attack (EA) research: introductory cross-eye jammer investigation CSIR, Pretoria, RSA, Rep.5865-EAR00005 RPT Rev 2, May 2008.

[21] W. L. Stutzman and G. A. Thiele, Antenna Theory and Design, 2nd ed. New York: Wiley, 1998.

[22] D. D. Howard, "Radar target angular scintillation in tracking and guidance systems based on echo signal phase front distortion," in Proc. Nat. Eng. Conf., D. K. Barton, Ed., 1959, vol. 15.

[23] J. E. Lindsay, "Angular glint and the moving, rotating, complex radar target," IEEE Trans. Aerosp. Electron. Syst., vol. 4, no. 2, pp. 164-173, Mar. 1968.

[24] J. Wright, "Radar glint-A survey," Electromagn., vol. 4, no. 2, pp. 205-227, Jan. 1984.

\section{Electrical Properties of Rat Skin and Design of Implantable Antennas for Medical Wireless Telemetry}

Tutku Karacolak, Robert Cooper, and Erdem Topsakal

Abstract-The design and test is described of a small size dual band implantable antenna operating in Medical Implant Communications Service (MICS) (402 MHz-405 MHz) and Industrial, Scientific and Medical (ISM) $(2.4 \mathrm{GHz}-2.48 \mathrm{GHz})$ bands to be used in animal studies for medical research. The antenna is intended for wireless medical monitoring of the physiological parameters such as glucose, pressure, temperature, etc. First, the electrical properties $\left(\varepsilon_{r}\right.$ and $\left.\sigma\right)$ of skin samples from donor rats are measured at Mississippi State University's (MSU) College of Veterinary Medicine. A dual band antenna is then designed using an in-house finite element boundary integral solver in conjunction with particle swarm optimization algorithm. Finally, the antenna is tested using both skin-mimicking materials and real skin samples. The development details of the skin-mimicking materials are also given. Results regarding the $S_{11}$ and gain of the designed antenna are given and discussed in detail.

Index Terms-Implantable antenna, medical implant communications service (MICS) band, scientific and medical (ISM) band, skin-mimicking material.

\section{INTRODUCTION}

Animal models are widely used in medical research to develop new technologies to treat and cure various diseases. Although different animals are used for different studies, without a doubt, the rats are the most commonly used laboratory animals in medical research. Studies that are intended for monitoring of the physiological parameters such as temperature, blood pressure, glucose etc. require implantation of a wireless data telemetry unit in the interstitial fluid under the skin. The implantable RF unit is composed of an implantable antenna, a transceiver and chip with necessary circuit components, and a biosensor. Our main focus in this study is designing and testing of a dual band implantable antenna that can be used in an implantable telemetry unit for future animal studies.

Antennas have major role in implantable systems since they provide communication of the implant with the external equipment [1], [2]. However, implantable antenna design is quite challenging due to antenna size, impedance matching, low-power requirements, and biocompatibility with the body's physiology [3]-[8]. In addition, the host environment (biological tissue) adds significant complexity to the problem due to high losses. Thus far, implantable antenna designs have been limited to in silico and in vitro studies. A small size dual MICS (402-405 MHz) and ISM (2.4-2.48 GHz) band implantable antenna intended for continuous glucose monitoring applications was designed in [9] using measured electrical properties of the human skin [10], [11]. This dual band operation allows the power conservation by allowing the implanted device to stay in the sleep mode until an ISM wake-up signal was received. The antenna then utilizes the MICS band to transmit data to a base station located outside the tissue.

Manuscript received August 22, 2008; revised December 17, 2008. First published July 10, 2009; current version published September 02, 2009.

T. Karacolak and E. Topsakal are with the Department of Electrical and Computer Engineering, Mississippi State University, Mississippi State, MS 39762 USA (e-mail: tk57@msstate.edu; topsakal@ece.msstate.edu).

R. Cooper is with the College of Veterinary Medicine, Mississippi State University, Mississippi State, MS 39762 USA (e-mail: rcooper@cvm.msstate.edu).

Color versions of one or more of the figures in this communication are available online at http://ieeexplore.ieee.org.

Digital Object Identifier 10.1109/TAP.2009.2027197 